\title{
COMPLETE QUOTIENT BOOLEAN ALGEBRAS
}

\author{
AKIHIRO KANAMORI AND SAHARON SHELAH
}

\begin{abstract}
For $I$ a proper, countably complete ideal on the power set $\mathscr{P}(X)$ for some set $X$, can the quotient Boolean algebra $\mathscr{P}(X) / I$ be complete? We first show that, if the cardinality of $X$ is at least $\omega_{3}$, then having completeness implies the existence of an inner model with a measurable cardinal. A well-known situation that entails completeness is when the ideal $I$ is a (nontrivial) ideal over a cardinal $\kappa$ which is $\kappa^{+}$-saturated. The second author had established the sharp result that it is consistent by forcing to have such an ideal over $\kappa=\omega_{1}$ relative to the existence of a Woodin cardinal. Augmenting his proof by interlacing forcings that adjoin Boolean suprema, we establish, relative to the same large cardinal hypothesis, the consistency of: $2^{\omega_{1}}=\omega_{3}$ and there is an ideal ideal $I$ over $\omega_{1}$ such that $\mathscr{P}\left(\omega_{1}\right) / I$ is complete. (The cardinality assertion implies that there is no ideal over $\omega_{1}$ which is $\omega_{2}$-saturated, and so completeness of the Boolean algebra and saturation of the ideal has been separated.)
\end{abstract}

For $I$ a proper, countably complete ideal on $\mathscr{P}(X)$ for some set $X$, can the quotient Boolean algebra $\mathscr{P}(X) / I$ be complete? This question was raised by Sikorski [Si] in 1949. By a simple projection argument as for measurable cardinals, it can be assumed that $X$ is an uncountable cardinal $\kappa$, and that $I$ is a $\kappa$-complete ideal on $\mathscr{P}(\kappa)$ containing all singletons. In this paper we provide consequences from and consistency results about completeness. Throughout, $\kappa$ will denote an uncountable cardinal, and by an ideal over $\kappa$ we shall mean a proper, $\kappa$-complete ideal on $\mathscr{P}(\kappa)$ containing all singletons.

If $\kappa$ is a measurable cardinal and $I$ a prime ideal over $\kappa$, then of course $\mathscr{P}(\kappa) / I$ is complete, being the two-element Boolean algebra. The following theorem shows that completeness in itself has strong consistency strength:

Theorem A. If $\kappa \geq \omega_{3}$ and there is an ideal I over $\kappa$ such that $\mathscr{P}(\kappa) / I$ is complete, then there is an inner model with a measurable cardinal.

The restriction $\kappa \geq \omega_{3}$ is necessary for our proof.

There is a well-known situation in which completeness obtains. An ideal over $\kappa$ is $\lambda$-saturated iff whenever $\left\{X_{\alpha} \mid \alpha<\lambda\right\} \subseteq \mathscr{P}(\kappa)-I$, there are $\alpha<\beta<\lambda$ such that $X_{\alpha} \cap X_{\beta} \notin I$. Smith-Tarski [ST] established that if an ideal $I$ over $\kappa$ is $\kappa^{+}$-saturated, then $\mathscr{P}(\kappa) / I$ is complete. There are several consistency results about the existence of such ideals; modifying a sharp one due to the second author, we establish the following result separating completeness from saturation.

Received by the editors August 13, 1990 and, in revised form, January 28, 1994.

1991 Mathematics Subject Classification. Primary 03E35; Secondary 03E55.

The second author is partially supported by the U.S.-Israel Binational Science Foundation. This paper is \#390 in his bibliography. 
NS denotes the ideal over $\omega_{1}$ consisting of the nonstationary subsets; for an ideal $I$ over $\kappa$ and $A \subseteq \kappa, I \mid A$ denotes $\{X \subseteq \kappa \mid X \cap A \in I\}$, the restriction of $I$ to $A$; finally, for $S \subseteq \omega_{1}, \widetilde{S}$ denotes $\omega_{1}-S$.

Theorem B. Suppose that $\kappa$ is a Woodin cardinal, $\mathrm{CH}$ holds, and $S$ is a stationary, co-stationary subset of $\omega_{1}$. Then there is a cardinal-preserving forcing extension with no new reals satisfying: $2^{\omega_{1}}=\kappa$, and $I=\mathrm{NS} \mid \widetilde{S}$ is an ideal over $\omega_{1}$ such that $\mathscr{P}\left(\omega_{1}\right) / I$ is a complete Boolean algebra (and I is not $\omega_{2}$ saturated).

Of course, starting with a Woodin cardinal we can insure that $\mathrm{CH}$ also holds by carrying out a preliminary extension. Also, the statement has the same force if $S$ is replaced by $\widetilde{S}$, but the formulation is notationally convenient for the proof. That the $I$ in the resulting model cannot be $\omega_{2}$-saturated follows from the following well-known result of Ketonen [Ke]: If $\mathrm{CH}$ holds and there is an $\omega_{2}$-saturated ideal over $\omega_{1}$ then $2^{\omega_{1}}=\omega_{2}$. Adding a further layer of complexity to the proof of Theorem B, we also establish

Theorem C. Assume the hypotheses of Theorem B. Then there is a forcing extension with no new reals satisfying: $2^{\omega_{1}}=\omega_{3}=\kappa$, and $I=\mathrm{NS} \mid \widetilde{S}$ is an ideal over $\omega_{1}$ such that $\mathscr{P}\left(\omega_{1}\right) / I$ is a complete Boolean algebra (and I is not $\omega_{2}$-saturated).

It follows from 1.1(a) below that $I$ must be $\omega_{3}$-saturated. This then contributes to the theory of saturated ideals by establishing the relative consistency of $2^{\omega}=\omega_{1}, 2^{\omega_{1}}=\omega_{3}$, and the existence of an $\omega_{3}$-saturated ideal over $\omega_{1}$.

In $\S 1$ we derive some consequences of completeness and establish Theorem A. In $\S 2$ we indicate the modifications necessary to a previous proof of the second author to establish Theorem B. Finally in $\S 3$ we build on $\S 2$ to establish Theorem $\mathrm{C}$, providing iteration lemmas for iterated semiproper forcing with mixed supports. The main mathematical advances in this paper are due to the second author, based on speculations and prodding by the first.

\section{CONSEQuences of COMPLETENesS}

We first review the various concepts involved to affirm some notation: Let $I$ be an ideal over $\kappa$. Then $I^{+}=\mathscr{P}(\kappa)-I$, the "positive measure" sets with respect to $I$. For any $A \subseteq \kappa,[A]_{I}=\{B \subseteq \kappa \mid A \triangle B \in I\}$. The Boolean algebra $\mathscr{P}(\kappa) / I$ consists of the $[A]_{I}$ 's with the set-theoretic operations modulated by $I:[A]_{I} \vee[B]_{I}=[A \cup B]_{I},[A]_{I} \wedge[B]_{I}=[A \cap B]_{I},-[A]_{I}=[\kappa-A]_{I}, \mathbf{0}_{I}=[\varnothing]_{I}$, and $\mathbf{1}_{I}=[\kappa]_{I}$. The subscript $I$ will be suppressed when clear from the context. A Boolean algebra is complete iff least upper bounds exist for any collection of its members. $\mathscr{A}$ is an antichain with respect to $I$ iff $\mathscr{A} \subseteq I^{+}$yet whenever $A, B \in \mathscr{A}$ are distinct, $A \cap B \in I$. Thus, $I$ is $\lambda$-saturated iff every antichain with respect to $I$ has cardinality $<\lambda$. Also, $\mathscr{A}$ is a maximal antichain iff $\bigvee\{[A] \mid A \in \mathscr{A}\}=\mathbf{1}$ yet whenever $A, B \in \mathscr{A}$ are distinct, $[A] \wedge[B]=\mathbf{0}$.

The first significant results bearing on Sikorski's question were derived by Solovay [So], who established the consistency, relative to the existence of a measurable cardinal, of the existence of saturated ideals over accessible cardinals. In passing, he in effect noted the following partial converse to the Smith-Tarski result: 
1.1. Lemma. Suppose that $I$ is an ideal over $\kappa$ such that $\mathscr{P}(\kappa) / I$ is complete. For any $\lambda$, if $I$ is not $\lambda$-saturated, then $2^{\lambda} \leq 2^{\kappa}$. In particular,

(a) $I$ is $2^{\kappa}$-saturated.

(b) If $2^{\kappa}<2^{\kappa^{+}}$, then I is $\kappa^{+}$-saturated.

Proof. Let $\left\langle A_{\alpha} \mid \alpha<\lambda\right\rangle$ enumerate (without repetitions) an antichain with respect to $I$. For any $X \subseteq \lambda$, let $a_{X}=\bigvee\left\{\left[A_{\alpha}\right] \mid \alpha \in X\right\}$. Then $X \neq Y$ implies that $a_{X} \neq a_{Y}$. Hence, $2^{\bar{\lambda}} \leq|\mathscr{P}(\kappa) / I| \leq 2^{\kappa}$.

Kunen established that if there is a $\kappa^{+}$-saturated ideal over $\kappa$, then $\kappa$ is measurable in an inner model. In particular, as Solovay noted, 1.1(b) implies that if there is an ideal $I$ over $\kappa$ such that $\mathscr{P}(\kappa) / I$ is complete, then $V \neq L$. Kunen asked in the early 1970s whether completeness has strong consistency strength, and Theorem A confirms this, at least if $\kappa \geq \omega_{3}$.

With our ultimate goal the proof of Theorem A, we now fix an ideal $I$ over $\kappa$ such that $\mathscr{P}(\kappa) / I$ is complete for the rest of this section. We use the well-known stratagem of considering $\mathscr{P}(\kappa) / I-\{0\}$ as a notion of forcing with $[X] \leq[Y]$ iff $X-Y \in I$, and we denote the corresponding forcing relation simply by $\Vdash$. Note that if $\left\langle A_{\alpha} \mid \alpha<\gamma\right\rangle$ enumerates without repetitions a maximal antichain with respect to $I$, then it corresponds to a name $\dot{\tau}$ for an ordinal specified by: $\left[A_{\alpha}\right] \Vdash \dot{\tau}=\beta$ iff $\alpha=\beta$.

The following lemmata derive consequences of completeness using maximal antichains.

1.2. Lemma. Suppose that $\gamma \leq \kappa$ and $i$ is a name such that $i \in \gamma$. Then there is a partition $\left\{B_{\xi} \mid \xi<\gamma\right\}$ of $\kappa$ such that: if $B_{\xi} \in I^{+}$, then $\left[B_{\xi}\right] \Vdash \dot{\tau}=\xi$.

Proof. Let $\mathscr{A}$ be a maximal antichain with respect to $I$ such that for any $A \in \mathscr{A}$, there is a $\xi_{A}<\gamma$ such that $[A] \Vdash \dot{\tau}=\xi_{A}$. By completeness, let $A_{\xi} \subseteq \kappa$ for $\xi<\gamma$ be such that $\left[A_{\xi}\right]=\bigvee\left\{[A] \mid \xi_{A}=\xi\right\}$. Then set $B_{\xi}=A_{\xi}-\bigcup_{\zeta<\xi} A_{\zeta}$ for $\xi<\gamma$, so that $\left[B_{\xi}\right]=\left[A_{\xi}\right]$ by $\kappa$-completeness. The $B_{\xi}$ 's are as required, once they are slightly modified to constitute a partition of all of $\kappa$.

\subsection{Lemma. Forcing with $\Vdash$ preserves all cardinals $<\kappa$.}

Proof. It suffices to show that if $\gamma<\kappa$ is regular, $\delta<\gamma$, and $\Vdash i$ : $\delta \rightarrow \gamma$, then $\Vdash \exists \eta<\gamma\left(\dot{\tau}^{*} \delta \subseteq \eta\right)$. For each $\beta<\delta$, let $\left\langle B_{\xi}^{\beta} \mid \xi<\gamma\right\rangle$ satisfy 1.2 for $\dot{\tau}(\beta)$. For each $\alpha<\kappa$, set

$$
\eta_{\alpha}=\sup \left\{\xi<\gamma \mid \alpha \in B_{\xi}^{\beta} \text { and } \beta<\delta\right\},
$$

so that $\eta_{\alpha}<\gamma$ by the regularity of $\gamma$. Next, set $E_{\eta}=\left\{\alpha<\kappa \mid \eta_{\alpha}=\eta\right\}$ for $\eta<\gamma$. Then $\bigcup_{\eta<\gamma} E_{\eta}=\kappa$ is a partition. Consequently, for any $X \in I^{+}$there is an $\eta<\gamma$ such that $E_{\eta} \cap X \in I^{+}$by $\kappa$-completeness and $\left[E_{\eta} \cap X\right] \Vdash \dot{\tau}^{\text {“ }} \delta \subseteq \eta$.

The proof of the following proposition is similar; it will not be needed in the rest of the paper. The only early result about complete quotient Boolean algebras other than the Smith-Tarski result, it appeared in terms of distributivity properties in Pierce [P], which also contained a similar formulation of the easy forcing fact that a notion of forcing adjoins a new function: $\lambda \rightarrow \lambda$ exactly when it adjoins a new subset of $\lambda$.

1.4. Proposition. Suppose that $2^{\nu}<\kappa$. Then forcing with $\Vdash$ does not adjoin any new functions: $\nu \rightarrow 2^{\nu}$. 
Proof. Suppose $\Vdash i: \nu \rightarrow 2^{\nu}$. For each $\beta<\nu$, let $\left\langle B_{\xi}^{\beta} \mid \xi<2^{\nu}\right\rangle$ be as in 1.2 with its $\gamma=2^{\nu}$ and its $\dot{\tau}=\dot{\tau}(\beta)$. For each $f: \nu \rightarrow 2^{\nu}$, let $E_{f}=\bigcap_{\beta<\nu} B_{f(\beta)}^{\beta}$. Then $\bigcup_{f} E_{f}=\kappa$ is a partition. Consequently, for any $X \in I^{+}$, there is an $f$ such that $E_{f} \cap X \in I^{+}$by $\kappa$-completeness, and $\left[E_{f} \cap X\right] \Vdash \dot{\tau}=\check{f}$.

The connection with inner models of measurability is made through the wellknown concept of precipitous ideal, due to Jech and Prikry. For an ideal $J$ over $\lambda$, if $G$ is generic over $V$ for the corresponding notion of forcing $\mathscr{P}(\lambda) / J-$ $\left\{0_{J}\right\}$, then $\{X \subseteq \lambda \mid[X] \in G\}$ is an ultrafilter on $\mathscr{P}(\lambda) \cap V$, and for any class $A$ in the sense of $V$, the ultrapower of $A$ with respect to this ultrafilter using functions in ${ }^{\lambda} V \cap V$ is called the generic ultrapower of $A$ by $G$. In this situation, if $\dot{\tau}$ is a name for a function in ${ }^{\lambda} A \cap V$, we denote by $(\dot{\tau})$ the name of the equivalence class of $i$ in the ultrapower. $J$ is precipitous iff for any such $G$, the generic ultrapower of $V$ by $G$ is well-founded. As Jech and Prikry showed, if there is a precipitous ideal $J$ over $\lambda$, then $\lambda$ is measurable in an inner model.

Before we turn to the proof of Theorem A, we establish a partial wellfoundedness result about generic ultrapowers. Continuing to work with our fixed ideal $I$ over $\kappa$ such that $\mathscr{P}(\kappa) / I-\{0\}$ is complete and the corresponding notion for forcing $\Vdash$, we first make an observation related to 1.2.

1.5. Lemma. Suppose that $\gamma<\kappa$ and $[X] \Vdash \dot{\tau}: \kappa \rightarrow \gamma \wedge \dot{\tau} \in \check{V}$. Then there is an $f: \kappa \rightarrow \gamma$ such that $[X] \Vdash(\dot{\tau})=(\check{f})$; i.e. $[X]$ forces that $\dot{\tau}$ and $\check{f}$ belong to the same equivalence class in the generic ultrapower.

Proof. For any set $x$, let $c_{x}$ denote the constant function: $\kappa \rightarrow\{x\}$. We first note that for any $[Y] \leq[X]$, there is a $[Z] \leq[Y]$ and a $\xi<\gamma$ such that $[Z] \Vdash(\dot{\tau})=\left(\check{c}_{\xi}\right)$. (There is a $[\bar{Y}] \leq[Y]$ and a $g: \kappa \rightarrow \gamma$ such that $[\bar{Y}] \Vdash \dot{\tau}=\check{g}$. By $\kappa$-completeness there is a $\xi<\gamma$ such that $Z=\{i \in \bar{Y} \mid g(i)=\xi\} \in I^{+}$. Thus, $[Z] \Vdash(\dot{\tau})=\left(\check{c}_{\xi}\right)$ by definition of generic ultrapower.)

Using this, let $\mathscr{A} \subseteq \mathscr{P}(X) \cap I^{+}$be a maximal antichain in $\mathscr{P}(X) \cap I^{+}$ such that for each $A \in \mathscr{A}$, there is a $\xi_{A}<\gamma$ with $[A] \Vdash(\dot{\tau})=\left(\check{c}_{\xi_{A}}\right)$. By completeness, let $A_{\xi} \subseteq X$ for $\xi<\gamma$ be such that $\left[A_{\xi}\right]=\bigvee\left\{[A] \mid \xi_{A}=\xi\right\}$. Then as in the proof of 1.2 , let $B_{\xi} \subseteq X$ for $\xi<\gamma$ be such that $\left[B_{\xi}\right]=\left[A_{\xi}\right]$ and $\left\{B_{\xi} \mid \xi<\gamma\right\}$ is a partition of $X$. Finally, define $f: \kappa \rightarrow \gamma$ by $f(i)=\xi$ iff $i \in B_{\xi}$ (and $f(i)=0$ if $\left.i \notin X\right)$. Then $[X] \Vdash(\dot{\tau})=(\check{f})$ by definition of generic ultrapower.

This leads to

1.6. Proposition. If $\kappa$ is a successor cardinal, then $\Vdash$ "the generic ultrapower of $\kappa$ is well-founded."

Remark. It follows from the conclusion that e.g. $\kappa$ is inaccessible in $L$.

Proof. Suppose that $\kappa=\mu^{+}$, and assume to the contrary that for some $X \in$ $I^{+},[X] \Vdash$ " $\left\langle\left(\dot{\tau}_{n}\right) \mid n \in \omega\right\rangle$ is an infinite descending sequence with $\dot{\tau}_{n} \in{ }^{\kappa} \kappa \cap \check{V}$ for $n \in \omega "$. We can assume that $[X] \Vdash\left(\dot{\tau}_{0}\right)=\left(\check{g}_{0}\right)$ for some $g \in{ }^{\kappa} \kappa$, and that $[X] \Vdash \forall n \in \omega \forall \xi<\kappa\left(\dot{\tau}_{n}(\xi) \leq \check{g}_{0}(\xi)\right)$.

For each $\alpha<\kappa$, let $e_{\alpha}: \alpha+1 \rightarrow \mu$ be an injection. Then let $\dot{\sigma}_{n}$ for $0<n<\omega$ be names such that $[X] \Vdash \forall \xi<\kappa\left(\dot{\sigma}_{n}(\xi)=\check{e}_{g_{0}(\xi)}\left(\dot{\tau}_{n}(\xi)\right)\right)$. Thus, 
$[X] \Vdash \dot{\sigma}_{n}: \kappa \rightarrow \mu$. By 1.5 , let $f_{n}: \kappa \rightarrow \mu$ for $n \in \omega$ be such that $[X] \Vdash\left(\dot{\sigma}_{n}\right)=$ $\left(\check{f}_{n}\right)$. Next, define $g_{n}: \kappa \rightarrow \kappa$ for $0<n<\omega$ by: $g_{n}(i)$ is the unique $\beta$ such that $f_{n}(i)=e_{g_{0}(\xi)}(\beta)$ if $f_{n}(i)$ is in the range of $e_{g_{0}(\xi)}$, and $=0$ otherwise. Then for each $n \in \omega,[X] \Vdash\left(\dot{\tau}_{n}\right)=\left(\check{g}_{n}\right)$. Finally, if $T_{n}=\left\{i \in \kappa \mid g_{n+1}(i)<g_{n}(i)\right\}$ for $n \in \omega$, then $[X] \leq\left[T_{n}\right]$ by our assumption on $[X]$ and the definition of generic ultrapower. Hence, $X \cap \bigcap_{n} T_{n}$ is not empty, but any $i$ in this set gives rise to an infinite descending set of ordinals $g_{0}(i)>g_{1}(i)>g_{2}(i) \cdots$, which is a contradiction.

Turning to the proof of Theorem A, we need another ingredient. The DoddJensen Covering Theorem for their inner model $K$ asserts that if there is no inner model with a measurable cardinal, then for any uncountable set $x$ of ordinals there is a set $y \supseteq x$ such that $y \in K$ and $|y|=|x|$. The definable class $K$ is extensionally preserved in all forcing extensions.

Proof of Theorem A. We take $\kappa \geq \omega_{3}$. Assume to the contrary that there is no inner model with a measurable cardinal. Suppose briefly that $G$ is generic

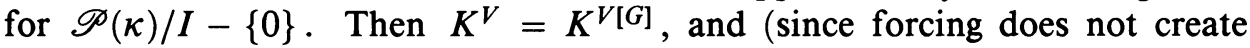
inner models of measurability) the conclusion of the Covering Theorem holds in $V[G]$. Since $\kappa \geq \omega_{3}$ and $\omega_{1}$ and $\omega_{2}$ are preserved by the forcing by 1.3 , this implies in particular: whenever $x$ is a size $\omega_{1}$ set of ordinals in $V[G]$, there is a $y \in V$ of size $\omega_{1}$ such that $y \supseteq x$. (Note that the preservation of $\omega_{2}$ is needed here.) Recalling a previous remark about maximal antichains corresponding to names for ordinals, this in turn translates in a straightforward manner to the following assertion in the ground model:

Whenever $\left\langle\mathscr{A}_{\alpha} \mid \alpha<\omega_{1}\right\rangle$ is a sequence of maximal antichains with respect to $I$, for any $X \in I^{+}$there is a $Y \in \mathscr{P}(X) \cap I^{+}$ such that for any $\alpha<\omega_{1},\left|\left\{A \in \mathscr{A}_{\alpha} \mid A \cap Y \in I^{+}\right\}\right| \leq \omega_{1}$.

We now derive a contradiction by using this to show that $I$ must be precipitous. Suppose then that $[X] \Vdash "\left\langle\left(\dot{\tau}_{n}\right) \mid n \in \omega\right\rangle$ is an infinite descending sequence in the generic ultrapower". For each $n \in \omega$, let $\mathscr{A}_{n}$ be a maximal antichain with respect to $I$ such that whenever $A \in \mathscr{A}_{n}$ there is an $f_{n}^{A}: \kappa \rightarrow V$ such that $[A] \Vdash \dot{\tau}_{n}=\check{f}_{n}^{A}$. Let $Y \in \mathscr{P}(X) \cap I^{+}$satisfy $(*)$ for $\left\langle\mathscr{A}_{n} \mid n \in \omega\right\rangle$ and for each $n \in \omega$ let $\left\langle A_{\xi}^{n} \mid \xi<\omega_{1}\right\rangle$ enumerate $\left\{A \in \mathscr{A}_{n} \mid A \cap Y \in I^{+}\right\}$. Set $B_{\xi}^{n}=\left(A_{\xi}^{n}-\bigcup_{\zeta<\xi} A_{\zeta}^{n}\right) \cap Y$ and $B_{n}=\bigcup_{\xi<\omega_{1}} B_{\xi}^{n}$, and define $f_{n}$ on $B_{n}$ by: $f_{n}=\bigcup_{\xi<\omega_{1}} f_{n}^{A_{\xi}^{n}} \mid B_{\xi}^{n}$. It is easily seen that for $n \in \omega,\left[B_{n}\right]=[Y]$ by maximality of $\mathscr{A}_{n}$ and $\kappa$-completeness. Hence $\bigcap_{n} B_{n} \neq \varnothing$, again by $\kappa$-completeness. But for any $i$ in this set, $f_{0}(i)>f_{1}(i)>\cdots$ which is a contradiction.

Properties of the sort $(*)$ were first investigated in Baumgartner-Taylor [BT], then in Foreman-Magidor-Shelah [FMS], by Woodin, and extensively in GitikShelah [GS]. A positive answer to the following question would strengthen 1.6 and eliminate the condition $\kappa \geq \omega_{3}$ from Theorem $\mathrm{A}$.

1.7. Question. If $\kappa$ is a successor cardinal and $I$ is an ideal over $\kappa$ such that $\mathscr{P}(\kappa) / I$ is complete, is $I$ precipitous? 


\section{SEPARATING COMPLETENESS FROM SATURATION}

We next turn to the proof of Theorem B. We shall build on the proof of the following result of Shelah [Sh4, XVI, Theorem 2.4], which we first describe.

2.1. Theorem. Suppose that $\kappa$ satisfies $(*)_{a b}^{a}$ (see [Sh4, XVI, 2.2]), e.g. is a Woodin cardinal, and $S$ is a stationary, co-stationary subset of $\omega_{1}$. Then there is a forcing extension with no new reals satisfying: $\kappa=\omega_{2}$ and $\mathrm{NS} \mid \widetilde{S}$ is an ideal over $\omega_{1}$ which is $\omega_{2}$-saturated.

This result weakened the large cardinal hypotheses of previous results drawing the same conclusion, and was an outgrowth of Foreman-Magidor-Shelah [FMS]. It was established using concepts and techniques of the second author that we quickly review:

Suppose that $\langle P, \leq\rangle$ is a poset for forcing, $\lambda$ is regular with $\mathscr{P}(P) \subseteq H_{\lambda}$, and $N$ is countable with $\langle N, \in\rangle \prec\left\langle H_{\lambda}, \epsilon\right\rangle$. Then $q \in P$ is $\langle N, P\rangle$-generic iff for any $P$-name $\dot{\tau} \in N$ for an ordinal, $q \Vdash i \in \check{\tau}$. Refining this, $q \in P$ is $\langle N, P\rangle$ semigeneric iff for any $P$-name $\dot{\tau} \in N$ for a countable ordinal, $q \Vdash \ddot{\tau} \in \check{N}$. $\left\langle p_{n} \mid n \in \omega\right\rangle$ is a P-generic sequence for $N$ iff $p_{n+1} \leq p_{n} \in N$ for each $n$ and whenever $D$ is a dense set for $P$ with $D \in N$, there is an $n$ such that $p_{n} \in D$.

$\langle P, \leq\rangle$ is semiproper iff for any regular $\lambda$ such that $\mathscr{P}(P) \subseteq H_{\lambda}$, there is a closed unbounded subset of $\left[H_{\lambda}\right]^{<\omega_{1}}$ consisting of $N$ such that $\langle N, \epsilon\rangle \prec$ $\left\langle H_{\lambda}, \in\right\rangle$ satisfying: for any $p \in N$, there is a $q \leq p$ such that $q$ is $\langle N, P\rangle$ semigeneric. For $S$ a stationary subset of $\omega_{1},\langle P, \leq\rangle$ is $S$-closed iff for any regular $\lambda$ such that $\mathscr{P}(P) \subseteq H_{\lambda}$, countable $N$ with $\langle N, \epsilon\rangle \prec\left(H_{\lambda}, \epsilon\right)$ and $N \cap \omega_{1} \in S$, and $P$-generic sequence $\left\langle p_{n} \mid n \in \omega\right\rangle$ for $N$, there is a $q \leq p_{n}$ for every $n \in \omega$. Semiproper is $\left\{\aleph_{1}\right\}$-semiproper and $S$-closed is $\{S\}$-complete in the sense of Shelah [Sh2]. $\omega_{1}$-closure readily implies semiproperness. The salient features of these concepts are that if a poset is semiproper, then forcing with it preserves stationary subsets of $\omega_{1}$ (i.e. any stationary subset of $\omega_{1}$ in the ground model remains stationary in the extension), and if it is $S$-closed, then it adjoins no new countable sequences of ordinals.

To affirm notation, $P$ is the countable support iteration of $\left\langle P_{\alpha}, \dot{Q}_{\alpha} \mid \alpha<\gamma\right\rangle$ iff setting $P_{\gamma}=P$, we have: $P_{0}=\{\varnothing\}$; for $\alpha<\gamma, \mathbb{H}_{P_{\alpha}}$ “ $\dot{Q}_{\alpha}$ is a poset" and $P_{\alpha+1}=P_{\alpha} * \dot{Q}_{\alpha} ;$ and for limit $\alpha \leq \gamma, P_{\alpha}$ is the direct limit of $\left\langle P_{\beta} \mid \beta<\alpha\right\rangle$ in case $\operatorname{cf}(\alpha)>\omega$, and the inverse limit otherwise. Proceeding recursively we can take $P_{\alpha}$ to consist of functions $p$ (the conditions) with domain $\alpha$ so that for each $\beta<\alpha, p(\beta)$ is a $P_{\beta}$-name and $\Vdash_{P_{\beta}} p(\beta) \in \dot{Q}_{\beta}$, and supposing that $\Vdash_{P_{\beta}}$ “ $\dot{1}_{Q_{\beta}}$ is the maximum element of $\dot{Q}_{\beta}$ ",

$$
\operatorname{supt}(p)=\left\{\beta<\alpha \mid \Vdash_{P_{\beta}} p(\beta) \neq \dot{\mathrm{i}}_{Q_{\beta}}\right\}
$$

is countable, with corresponding partial order on $P_{\alpha}$ given by: $p \leq q$ iff $\forall \beta<$ $\alpha\left(p \mid \beta \Vdash_{P_{\beta}} p(\beta) \leq q(\beta)\right)$.

Throughout the paper we rely on the following convention: For a notion of forcing $P, \dot{G}_{P}$ denotes the canonical $P$-name for its generic object, and if $P=P_{\alpha}$ in some contextually clear indexing, $\dot{G}_{\alpha}$ is written for $\dot{G}_{P_{\alpha}}$.

2.2. Lemma. Suppose $P$ is the countable support iteration of $\left\langle P_{\alpha}, \dot{Q}_{\alpha} \mid \alpha<\gamma\right\rangle$ where for $\alpha<\gamma, \mathbb{1}_{P_{x}}$ " $\dot{Q}_{\alpha}$ is semiproper and $S$-closed". Then $P$ is semiproper and S-closed. 
This is a special case of more general iteration lemmas. The appropriate mode of iteration for semiproperness is revised countable support (RCS) iteration, but $S$-closure at each stage implies that there are no new countable sequences of ordinals, and so RCS iteration reduces to countable support iteration.

In [FMS], 2.1 is established for $\kappa$ supercompact instead of, e.g. Woodin, by first establishing the consistency with $\mathrm{CH}$ of a Martin's Axiom for $S$-closed notions of forcing that preserve stationary subsets of $\omega_{1}$ and meeting $\omega_{1}$ dense sets. Then it suffices to argue with a notion of forcing which in the formulation of Shelah [Sh3] is as follows:

Suppose that $\mathscr{A}$ consists of stationary subsets of $\omega_{1}$ with $S \in \mathscr{A}$. Then $\operatorname{Seal}_{S}(\mathscr{A})$ consists of countable sequences $\left\langle N_{\xi} \mid \xi \leq \gamma\right\rangle$ such that:

(i) each $N_{\xi} \in\left[H_{\lambda}\right]^{<\omega_{1}}$, where $\lambda=\left(2^{\omega_{1}}\right)^{+}$, and $S \in N_{0}$.

(ii) $\left\langle N_{\xi} \mid \xi \leq \gamma\right\rangle$ is increasing and continuous.

(iii) for each $\xi \leq \gamma, N_{\xi} \cap \omega_{1}$ is an ordinal in $\bigcup\left\{A \mid A \in \mathscr{A} \cap N_{\xi}\right\}$.

$\operatorname{Seal}_{S}(\mathscr{A})$ is ordered by: $p \leq q$ iff $q$ is an initial segment of $p$.

$\operatorname{Seal}_{S}(\mathscr{A})$ is clearly $S$-closed, although it may not be semiproper, and forcing with it provides an enumeration of $\mathscr{A}$ in ordertype $\leq \omega_{1}$ and a closed unbounded subset of the diagonal union of $\mathscr{A}$ according to that enumeration. If $\mathscr{A}$ was a maximal antichain with respect to NS, then it can be shown that forcing with $\operatorname{Seal}_{S}(\mathscr{A})$ preserves stationary subsets of $\omega_{1} . \mathscr{A}$ is then "sealed": it remains a maximal antichain in any extension that preserves stationary sets, since any stationary set has stationary intersection with the diagonal union of $\mathscr{A}$, and hence with a particular member of $\mathscr{A}$ by Fodor's Lemma. The aforementioned version of Martin's Axiom implied through this means that NS $\mid \widetilde{S}$ is $\omega_{2}$-saturated.

2.1 was established by applying reflection properties directly in [Sh4, XVI]. Let

$$
Q_{S}=\left(\prod\left\{\operatorname{Seal}_{S}(\mathscr{A}) \mid \operatorname{Seal}_{S}(\mathscr{A}) \text { is semiproper }\right\}\right) * \operatorname{col}\left(\omega_{1}, 2^{\omega_{1}}\right),
$$

the countable support product of all $\operatorname{Seal}_{S}(\mathscr{A})$ 's for $\mathscr{A}$ 's that yield semiproper $\operatorname{Seal}_{S}(\mathscr{A})$, followed by the usual collapse of $2^{\omega_{1}}$ to $\omega_{1}$ using countable approximations. Clearly $Q_{S}$ is $S$-closed; it is also semiproper (see [Sh4, XIII, 2.8(3)] or [Sh3, 2.8(3), p. 361]). Let $P$ be the countable support iteration of $\left\langle P_{\alpha}, \dot{Q}_{\alpha} \mid \alpha<\kappa\right\rangle$ where $\dot{Q}_{\alpha}$ is a $P_{\alpha}$-name for $Q_{S}$ in the sense of $V^{P_{\alpha}}$. Assuming that $\kappa$ satisfies the large cardinal hypothesis of 2.1 , the second author showed that any forcing extension via $P$ satisfies its conclusion.

Proof of Theorem B. We interlace into the above described proof of 2.1 natural notions of forcing for introducing suprema into $\mathscr{P}\left(\omega_{1}\right) / \mathrm{NS} \mid \widetilde{S}:\langle\mathscr{A}, \mathscr{B}\rangle$ is an appropriate pair if $\mathscr{A}$ and $\mathscr{B}$ consist of subsets of $\omega_{1}$ such that: if $A \in \mathscr{A}$ and $B \in \mathscr{B}$, then $A \cap B \cap \widetilde{S}$ is nonstationary. For such $\langle\mathscr{A}, \mathscr{B}\rangle$, let $\operatorname{Sup}_{S}(\mathscr{A}, \mathscr{B})$ consist of triples $\langle w, c, d\rangle$ such that:

(i) $w$ is a countable subset of $\mathscr{A} \cup \mathscr{B}$.

(ii) $c$ is a countable, closed set of countable ordinals (so $\bigcup c \in c$ ).

(iii) $d: c \rightarrow 2$.

Order $\operatorname{Sup}_{S}(\mathscr{A}, \mathscr{B})$ by: $\langle w, c, d\rangle \leq\langle\bar{w}, \bar{c}, \bar{d}\rangle$ iff $\bar{w} \subseteq w, \bar{c}$ is an initial 
segment of $c, \bar{d} \subseteq d$, and:

if $\alpha \in(c \sim \bar{c}) \cap \widetilde{S}$ and $d(\alpha)=0$, then $\alpha \notin A$ for any $A \in \bar{w} \cap \mathscr{A}$.

if $\alpha \in(c \sim \bar{c}) \cap \widetilde{S}$ and $d(\alpha)=1$, then $\alpha \notin B$ for any $B \in \bar{w} \cap \mathscr{B}$.

Suppose that $G$ is $\operatorname{Sup}_{S}(\mathscr{A}, \mathscr{B})$-generic and set $c_{G}=\bigcup\{c \mid\langle w, c, d\rangle \in G\}$ and $d_{G}=\bigcup\{d \mid\langle w, c, d\rangle \in G\}$. Then $c_{G}$ is a closed unbounded subset of $\omega_{1}, d_{G}^{-1}(\{0\}) \cap A \cap \widetilde{S}$ is countable for every $A \in \mathscr{A}$, and $d_{G}^{-1}(\{1\}) \cap B \cap \widetilde{S}$ is countable for every $B \in \mathscr{B}$. In particular, if $\mathscr{A} \cup \mathscr{B}$ were a maximal antichain with respect to $\mathrm{NS} \mid \widetilde{S}$, then $\left[d_{G}^{-1}(\{1\})\right]$ would serve as an upper bound of $\mathscr{A}$ and $\left[d_{G}^{-1}(\{0\})\right]$ of $\mathscr{B}$. These will be least upper bounds in the extension if $\mathscr{A} \cup \mathscr{B}$ continues to be maximal there, and this is the only situation that will be germane to the overall argument. Of course, for all this to make sense in the extension we must ascertain that stationary subsets of $\omega_{1}$ are preserved:

\subsection{Lemma.}

(a) $\operatorname{Sup}_{S}(\mathscr{A}, \mathscr{B})$ is $\omega_{1}$-closed (and hence semiproper and $S$-closed).

(b) Assuming $\mathrm{CH}, \operatorname{Sup}_{S}(\mathscr{A}, \mathscr{B})$ is $\omega_{1}$-linked with least upper bounds; i.e. it is the union of $\omega_{1}$ sets, each consisting of pairwise compatible elements with least upper bounds.

Proof. (a) Set $P=\operatorname{Sup}_{S}(\mathscr{A}, \mathscr{B})$. Suppose that $\lambda$ is regular with $\mathscr{P}(P) \subseteq H_{\lambda}$, $N$ is countable with $\langle N, \in\rangle \prec\left\langle H_{\lambda}, \epsilon\right\rangle$ and $\left\langle p_{n} \mid n \in \omega\right\rangle$ is a $P$-generic sequence for $N$. We must find a $q \in P$ such that $q \leq p_{n}$ for every $n \in \omega$.

For $n \in \omega$ and $p_{n}=\left\langle w_{n}, c_{n}, d_{n}\right\rangle$, since $w_{n}$ is countable, we can let $C_{n} \in N$ be a closed unbounded subset of $\omega_{1}$ such that for any $A \in w_{n} \cap \mathscr{A}$ and $B \in w_{n} \cap \mathscr{B}, C_{n} \cap A \cap B \cap \widetilde{S}=\varnothing$. Set $w=\bigcup w_{n}, c=\bigcup c_{n}, d=\bigcup d_{n}$. Then a simple genericity argument implies that $\bigcup c \in \cap C_{n}$ so that for no $A \in w \cap \mathscr{A}$ and $B \in w \cap \mathscr{B}$ does $\bigcup c \in A \cap B$, and so we can find an $i<2$ such that $\langle w, c \cup\{\cup c\}, d \cup\{\langle\cup c, i\rangle\}\rangle$ is in $\operatorname{Sup}_{S}(\mathscr{A}, \mathscr{B})$ and of course is $\leq p_{n}$ for every $n \in \omega$.

(b) Note that $\left\langle w_{0}, c, d\right\rangle$ and $\left\langle w_{1}, c, d\right\rangle$ are compatible with least upper bound $\left\langle w_{0} \cup w_{1}, c, d\right\rangle$. With $\mathrm{CH}$, there are $\omega_{1}$ such pairs.

Let $\operatorname{Sup}_{S}$ be the countable support product of $\operatorname{Sup}_{S}(\mathscr{A}, \mathscr{B})$ for all appropriate pairs $\langle\mathscr{A}, \mathscr{B}\rangle$. A countable support product of $\omega_{1}$-closed forcings is readily seen to be $\omega_{1}$-closed, and $\omega_{1}$-closed forcings are $S$-closed and semiproper. Hence by 2.3(a), $\operatorname{Sup}_{S}$ is $S$-closed and semiproper.

An approach to the proof of Theorem B would be to carry out the countable support iteration of $\operatorname{Sup}_{S}$ through $\kappa$ stages. Just assuming $\operatorname{cf}(\kappa)>\omega_{1}$, we would then get the consistency of $2^{\omega_{1}}=\kappa$ and every appropriate pair $\langle\mathscr{A}, \mathscr{B}\rangle$ with $|\mathscr{A} \cup \mathscr{B}|<\kappa$ can be separated, a consequence in fact of a generalized Martin's Axiom in Baumgartner [B] or in Shelah [Sh1]. It is to ensure $\kappa$ saturation, a necessary condition for full completeness by $1.1(\mathrm{a})$, that we build on the proof of 2.1 .

Let $P_{\kappa}$ be the countable support iteration of $\left\langle P_{\alpha}, \dot{Q}_{\alpha} \mid \alpha<\kappa\right\rangle$, where:

(i) For odd $\alpha<\kappa, \dot{Q}_{\alpha}$ is a $P_{\alpha}$-name for $Q_{S}$ in the sense of $V^{P_{\alpha}}$. (Here, $Q_{S}$ is as in the above outline of the proof of 2.1.)

(ii) For even $\alpha<\kappa, \dot{Q}_{\alpha}$ is a $P_{\alpha}$-name for the countable support product of $\operatorname{Sup}_{S}(\mathscr{A}, \mathscr{B})$ 's for all appropriate pairs $\langle\mathscr{A}, \mathscr{B}\rangle$ satisfying $\langle\mathscr{A}, \mathscr{B}\rangle \in$ 
$V\left[\dot{G}_{\alpha} \mid\{2 \gamma \mid 2 \gamma<\alpha\}\right]$. (For $Z \subseteq \alpha, \dot{G}_{\alpha} \mid Z$ is the $P_{\alpha}$-name for $\left\{p \in \dot{G}_{\alpha} \mid \operatorname{supt}(p) \subseteq\right.$ $Z$ \}. Note that $\langle\mathscr{A}, \mathscr{B}\rangle$ is to belong to the smaller model, but in the definition of "appropriate pair" the nonstationariness in " $(\forall A \in \mathscr{A})(\forall B \in \mathscr{B}) A \cap B \cap \widetilde{S}$ is not stationary" is to be in the sense of $\left.V\left[G_{\alpha}\right] !\right)$

Next, for $\alpha \leq \kappa$ set, by induction on $\alpha$ :

$$
P_{\alpha}^{\prime}=\left\{p \in P_{\alpha} \mid \operatorname{supt}(p) \subseteq\{2 \gamma \mid 2 \gamma<\alpha\} \wedge \forall \beta \in \operatorname{supt}(p)\left(p(\beta) \text { is a } P_{\beta}^{\prime} \text {-name }\right)\right\}
$$

with the inherited order. $\left(p(\beta)\right.$ being a $P_{\beta}^{\prime}$-name, it only depends on $\dot{G}_{\beta} \cap P_{\beta}^{\prime}$.) We show that any forcing extension via $P_{\kappa}^{\prime}$ satisfies the conclusion of Theorem B. This follows from the following technical lemma, all of whose parts are established by simultaneous induction; for its (b), note that $P_{\beta}^{\prime}$-names being $P_{\beta}$-names is justified by an inductive appeal to (a), and $\operatorname{Sup}_{S}$ was defined a few paragraphs ago in the outline of the proof of 2.1 .

2.4. Lemma. For each $\alpha \leq \kappa$ :

(a) $P_{\alpha}^{\prime} \otimes P_{\alpha}$; i.e. every maximal antichain of $P_{\alpha}^{\prime}$ is a maximal antichain of $P_{\alpha}$.

(b) $P_{\alpha}^{*}=\left\{p \in P_{\alpha} \mid \forall\right.$ even $\beta \in \operatorname{supt}(p)\left(p(\beta)\right.$ is a $P_{\beta}^{\prime}$-name for a condition in Sup $_{S}$ in the sense of $\left.V^{P_{\beta}^{\prime}}\right\}$ is dense in $P_{\alpha}$.

(c) For any $p, q \in P_{\alpha}^{*}$ such that $p \mid\{2 \gamma \mid 2 \gamma<\alpha\}$ and $q \mid\{2 \gamma \mid 2 \gamma<\alpha\}$ are compatible members of $P_{\alpha}^{\prime}$, there is an automorphism $F_{p q}^{\alpha}$ such that: $F_{p q}^{\alpha}(p)$ is compatible with $q ; F_{p q}^{\alpha}$ is the identity on $P_{\alpha}^{\prime}$; and inductively for any $\beta<\alpha$,

$$
F_{p|\beta, q| \beta}^{\beta}=F_{p q}^{\alpha} \mid P_{\beta}
$$

(d) If $p \in P_{\alpha}^{*}$, then $p \mid\{2 \gamma \mid 2 \gamma<\alpha\} \in P_{\alpha}^{\prime}$.

Once this lemma is established, the proof of Theorem B can be completed as follows: Suppose that $G^{\prime}$ is $P_{\kappa}^{\prime}$-generic, and by $2.4(\mathrm{a})$ let $G$ be $P_{\kappa}$-generic such that $G \cap P_{\kappa}^{\prime}=G^{\prime}$. Note first that for any $X \subseteq \omega_{1}$ with $X \in V\left[G^{\prime}\right]$,

$$
V\left[G^{\prime}\right] \Vdash X \text { is stationary iff } V[G] \Vdash X \text { is stationary. }
$$

(If $X$ were nonstationary in $V[G]$, then it would be nonstationary in $V\left[G \cap P_{\alpha}\right]$ for some even $\alpha<\kappa$. But then $X=A \cap B \cap \tilde{S}$ for some $A \in \mathscr{A}$ and $B \in \mathscr{B}$ with $\langle\mathscr{A}, \mathscr{B}\rangle$ an appropriate pair in $V\left[G \cap P_{\alpha}^{\prime}\right]$, so that $\operatorname{Sup}_{S}(\mathscr{A}, \mathscr{B})$ at that stage would have adjoined a closed unbounded subset of $\omega_{1}$ confirming that $X$ is nonstationary in $V\left[G^{\prime}\right]$.) The proof of 2.1 still works to show that in $V[G]$, $\kappa=\omega_{2}$ and $\mathrm{NS} \mid \widetilde{S}$ is $\kappa$-saturated. It thus follows that $\mathrm{NS} \mid \widetilde{S}$ is also $\kappa$-saturated in $V\left[G^{\prime}\right]$.

We can conclude that for any maximal antichain $\mathscr{A} \cup \mathscr{B}$ of $\mathrm{NS} \mid \widetilde{S}$ in the sense of $V\left[G^{\prime}\right]$ with $\mathscr{A} \cap \mathscr{B}=\varnothing,\langle\mathscr{A}, \mathscr{B}\rangle \in V\left[G^{\prime} \cap P_{\alpha}^{\prime}\right]$ for some even $\alpha<\kappa$. But then, the forcing with $\operatorname{Sup}_{S}(\mathscr{A}, \mathscr{B})$ would have adjoined a set $E \subseteq \omega_{1}$ such that: if $A \in \mathscr{A}$, then $A-(E \cap \widetilde{S})$ is not stationary; and if $B \in \mathscr{B}$, then $B \cap E \cap \widetilde{S}$ is not stationary. [ $E \cap \widetilde{S}]$ is thus the supremum for $\mathscr{A}$ in $V\left[G^{\prime} \cap P_{\alpha+1}^{\prime}\right]$ and hence in $V\left[G^{\prime}\right]$. This suffices to show that in $V\left[G^{\prime}\right], \mathscr{P}\left(\omega_{1}\right) / \mathrm{NS} \mid \widetilde{S}$ is a complete Boolean algebra.

Finally, $P_{\kappa}^{\prime}$ is $S$-closed, so that forcing with it adjoins no new countable sequences of ordinals. Consequently, $\left\{p \in P_{\kappa}^{\prime} \mid\right.$ if $\beta \in \operatorname{supt}(p)$ and $\langle\dot{w}, \dot{c}, \dot{d}\rangle$ 
is a component of $p(\beta)$, then $\dot{c}=\check{c}$ and $\dot{d}=\check{d}$ for some $c, d \in V\}$ is dense in $P_{\kappa}^{\prime}$. Using $\mathrm{CH}$ and 2.3(b), a simple $\Delta$-system argument using this dense set then shows that $P_{\kappa}^{\prime}$ has the $\omega_{2}$-c.c and hence preserves all cardinals, and it is simple to see that it renders $2^{\omega_{1}}=\kappa$.

Proof of 2.4. Assuming that $\alpha \leq \kappa$ and all four parts hold below $\alpha$, we verify that they all hold at $\alpha$.

To first verify that (b) holds at $\alpha$, suppose that $p \in P_{\alpha}$ is arbitrary. Let $\lambda$ be regular and sufficiently large, and $N$ countable such that $\langle N, \epsilon\rangle \prec\left(H_{\lambda}, \epsilon\right\rangle$, $p \in N$, and $N \cap \omega_{1} \in S$. Let $\left\langle p_{n} \mid n \in \omega\right\rangle$ be a $P_{\alpha}$-generic sequence for $N$ with $p_{0}=p$. Incorporating the proof of 2.3(a) into the iteration lemma for $S$-closed notions of forcing, there is a least upper bound $q \in P_{\alpha}$ for the $p_{n}$ 's specified as follows:

$\operatorname{supt}(q)=\bigcup_{n} \operatorname{supt}\left(p_{n}\right)$. For even $\beta \in \operatorname{supt}(q), q \mid \beta$ forces that for component $\langle\dot{w}, \dot{c}, \dot{d}\rangle$ of $q(\beta)$ in some relevant $\operatorname{Sup}_{S}(\dot{\mathscr{A}}, \dot{\mathscr{B}})$ with corresponding $\left\langle\dot{w}_{n}, \dot{c}_{n}, \dot{d}_{n}\right\rangle$ in $p_{n}$ for $n \in \omega$ sufficiently large, $\dot{w}$ is the union of the $\dot{w}_{n}$ 's, $\dot{c}$ is the union of the $\dot{c}_{n}$ 's together with its limit point at the top, and $\dot{d}$ is the union of the $\dot{d}_{n}$ 's together with an arbitrary value for that top limit point. By $P_{\alpha}$-genericity of $\left\langle p_{n} \mid n \in \omega\right\rangle$ and induction it can be assumed that each $\dot{w}_{n}$ is a $P_{\beta}^{\prime}$-name and hence that $\dot{w}$ is a $P_{\beta}^{\prime}$-name, and that $\dot{c}=\check{c}$ for some $c \in V$ and $\dot{d}=\check{d}$ for some $d \in V$.

Continuing to consider that specific component $\langle\dot{w}, \dot{c}, \dot{d}\rangle$ of $q(\beta)$, by definition of such conditions there is a $P_{\beta}^{\prime}$-name $\dot{w}_{0}$ such that $q \mid \beta \Vdash$ “ $\left\langle\dot{w} \cap \dot{w}_{0}, \dot{w}-\dot{w}_{0}\right\rangle$ is an appropriate pair". By induction, $P_{\beta}^{\prime} \odot P_{\beta}$, and the homogeneity property $2.4(\mathrm{c})$ implies that whenever $r \in P_{\beta}, \dot{\tau}$ is a $P_{\beta}^{\prime}$-name, $\psi$ is a one-free variable formula, and $r \Vdash_{P_{\beta}} \psi(\dot{\tau})$, then $r \mid\{2 \gamma \mid 2 \gamma<\beta\} \Vdash_{P_{\beta}^{\prime}} \psi(\dot{\tau})$. In particular

$$
(q \mid \beta) \mid\{2 \gamma \mid 2 \gamma<\beta\} \Vdash_{P_{\beta}^{\prime}} \text { “ }\left\langle\dot{w} \cap \dot{w}_{0}, \dot{w}-\dot{w}_{0}\right\rangle \text { is an appropriate pair”. }
$$

This confirms that $q \in P_{\alpha}^{*}$ to verify $2.4(\mathrm{~b})$ for $\alpha$ as desired.

At the referee's urging we also elaborate the rest:

To establish (d) at $\alpha$, let $p \in P_{\alpha}^{*}, \beta$ an even ordinal in $\operatorname{supt}(p)$, and set $p^{c}=p \mid\{2 \gamma \mid 2 \gamma<\beta\}$. It must be shown that $p^{c} \Vdash_{P_{\beta}} p(\beta) \in \dot{Q}_{\alpha}$. We know inductively that $P_{\beta}^{\prime} \varangle P_{\beta}, p^{c} \in P_{\beta}^{\prime}$, and $p^{c} \Vdash_{P_{\beta}^{\prime}} p(\beta) \in \dot{Q}_{\beta}$. The only problem in trying to replace $P_{\beta}^{\prime}$ by $P_{\beta}$ here is that an appropriate pair mentioned in $p(\beta)$ in the sense of $V^{P_{\beta}^{\prime}}$ may no longer be one in the sense of $V^{P_{\beta}}$.

Assume to the contrary that for some $q \in P_{\beta}$ with $q \leq p^{c}, q \Vdash_{P_{\beta}} \dot{p}(\beta) \notin$ $\dot{Q}_{\beta}$. By (b) inductively it can be assumed that $q \in P_{\beta}^{*}$, and by (d) inductively, $q \mid\{2 \gamma \mid 2 \gamma<\beta\} \in P_{\beta}^{\prime}$. Since $q \mid\{2 \gamma \mid 2 \gamma<\beta\} \leq p^{c}$ in $P_{\beta}^{\prime}$, there is an automorphism $F_{q, p \mid b}^{\beta}$ as in (c) inductively such that $F_{q, p \mid \beta}^{\beta}(q)$ is compatible with $p \mid \beta$. Hence, for some $q^{+} \in P_{\beta}$ with $q^{+} \leq q, F_{q, p \mid \beta}^{\beta}\left(q^{+}\right) \leq p \mid \beta$. Let $G \subseteq P_{\beta}$ be $P_{\beta}$-generic over $V$ with $q^{+} \in G$. Then $G^{\prime}=F_{q, p \mid \beta}^{\beta}$ " $G$ is $P_{\beta}$-generic over $V$ such that $p \mid \beta \in G^{\prime}$. But $G \cap P_{\beta}^{\prime}=G^{\prime} \cap P_{\beta}^{\prime}$ as $F_{q, p \mid \beta}^{\beta}$ is the identity on $P_{\beta}^{\prime}$, and 
so

$$
\begin{aligned}
& \left\{\langle\mathscr{A}, \mathscr{B}\rangle \mid \mathscr{A} \cup \mathscr{B} \subseteq \mathscr{P}\left(\omega_{1}\right) \cap V\left(G \cap P_{\beta}^{\prime}\right)\right\} \\
& \quad=\left\{\langle\mathscr{A}, \mathscr{B}\rangle \mid \mathscr{A} \cup \mathscr{B} \subseteq \mathscr{P}\left(\omega_{1}\right) \cap V\left[G^{\prime} \cap P_{\beta}^{\prime}\right]\right\},
\end{aligned}
$$

and so as $V[G]=V\left[G^{\prime}\right]$,

$$
\begin{aligned}
& \left\{\langle\mathscr{A}, \mathscr{B}\rangle \in V\left[G \cap P_{\beta}^{\prime}\right] \mid\langle\mathscr{A}, \mathscr{B}\rangle \text { is appropriate in } V[G]\right\} \\
& \left.\quad=\left\{\langle\mathscr{A}, \mathscr{B}\rangle \in V\left[G \cap P_{\beta}^{\prime}\right] \mid\langle\mathscr{A}, \mathscr{B}\rangle \text { is appropriate in } V\left[G^{\prime}\right]\right)\right\} .
\end{aligned}
$$

As also all $\omega$-sequence from $V$ of members of $V\left[G \cap P_{\beta}^{\prime}\right]$ are in $V\left[G \cap P_{\beta}^{\prime}\right]$ (as this holds for $V$ and $V[G])$, clearly $\left(\dot{Q}_{\beta}\right)^{G}=\left(\dot{Q}_{\beta}\right)^{G^{\prime}}$. This contradicts the choice of $q$.

Now clause (a) for $\alpha$ follows: For $p \in P_{\alpha}$, choose $q$ such that $q \leq p \in P_{\alpha}^{*}$. Setting $q^{c}=q \mid\{2 \gamma \mid 2 \gamma<\alpha\}, q^{c} \in P_{\alpha}^{\prime}$ by clause $(\mathrm{d})$ and $q^{c} \Vdash_{P_{\alpha}^{\prime}}$ " $p \in P_{\alpha} / \dot{G}_{P_{\alpha}^{\prime}}$ " because if $r \leq q^{c} \in P_{\alpha}^{\prime}$ then $r^{\prime}=r \cup(q \mid\{\beta<\alpha \mid \beta$ is odd $\})$ is in $P_{\alpha}^{*}$ (check!) and is below $r$ and $q$ hence below $p$.

We lastly deal with clause (c) for $\alpha$. If $\alpha$ is a limit, it is immediate: $F_{p, q}^{\alpha}(r)$ is defined by: $\gamma \in \operatorname{supt}\left(F_{p, q}^{\alpha}(r)\right)$ iff for some (equivalently, every) $\beta \in(\gamma, \alpha)$ we have $\gamma \in \operatorname{supt}\left(F_{p|\beta, q| \beta}^{\beta}(r)\right)$, and letting $\left(F_{p, q}^{\alpha}(r)\right)(\gamma)=\left(F_{p|\beta, q| \beta}^{\beta}(r)\right)(\gamma)$ for some (any) such $\beta$.

If $\alpha=\beta+1, \beta$ odd, just note that $\dot{Q}_{\beta}$ is definable in $V\left[\dot{G}_{P_{\beta}}\right.$ (without parameters). If $\alpha=\beta+1, \beta$ even, if $G \subseteq P_{\beta}$ is generic over $V$, in $V\left[G \cap P_{\beta}^{\prime}\right]$ there is an automorphism $F_{\beta}$ of $\dot{Q}_{\beta}[G]$ (note: $\dot{Q}_{\beta}[G] \in V\left[G \cap P_{\beta}^{\prime}\right]$-see the proof of clause $(\mathrm{d})$ ) such that: $F_{\beta}(p(\beta))$, and $q(\beta)$ are compatible. (The simplest way to see this is to replace in the iteration $\dot{Q}_{\alpha}$ by its completion.)

\section{Collapsing $\kappa$ to $\omega_{3}$}

In this section we specify the modifications to the proof of Theorem B necessary to establish Theorem $\mathrm{C}$. We define the components of an iteration $\left\langle P_{\alpha}, \dot{R}_{\alpha} \mid \alpha<\kappa\right\rangle$ in three cases instead of two:

(i) For $\alpha \equiv 0(\bmod 3), \dot{R}_{\alpha}$ is defined as $\dot{Q}_{\alpha}$ was before for odd $\alpha$; i.e. it is $Q_{S}^{V^{P_{\alpha}}}$

(ii) For $\alpha \equiv 1(\bmod 3), \dot{R}_{\alpha}$ is defined as $\dot{Q}_{\alpha}$ was before for even $\alpha$, but for all appropriate pairs $\langle\mathscr{A}, \mathscr{B}\rangle$ satisfying

$$
\langle\mathscr{A}, \mathscr{B}\rangle \in V\left[\dot{G}_{\alpha} \mid\{\beta<\alpha \mid \beta \equiv 1(\bmod 3) \vee \beta \equiv 2(\bmod 3)\}\right]
$$

(where $\dot{G}_{\alpha} \mid Z$ is as before).

(iii) For $\alpha \equiv 2(\bmod 3), \dot{R}_{\alpha}$ is $\operatorname{col}\left(\omega_{2}, 2^{\omega_{2}}\right)$, the collapse of $2^{\omega_{2}}$ to $\omega_{2}$ using $\omega_{1}$ size approximations, in the sense of $V\left[\dot{G}_{\alpha} \mid\{\beta<\alpha \mid \beta \equiv 1(\bmod 3) \vee\right.$ $\beta \equiv 2(\bmod 3)\}]$.

The latter notion of forcing is semiproper and $S$-closed, being $\omega_{1}$-closed. Its introduction necessitates that we define the $P_{\alpha}$ 's with mixed support: Proceeding recursively, for $\alpha \leq \kappa$ let $\bar{P}_{\alpha}$ consist of functions $p$ with domain $\alpha$ such that for each $\beta<\alpha, p(\beta)$ is a $\bar{P}_{\beta}$-name such that $\Vdash_{\bar{P}_{\beta}} p(\beta) \in \dot{R}_{\beta}$, and

$$
\begin{aligned}
& |\operatorname{supt}(p) \cap\{\beta<\alpha \mid \beta \equiv 0(\bmod 3) \vee \beta \equiv 1(\bmod 3)\}| \leq \aleph_{0}, \\
& |\operatorname{supt}(p) \cap\{\beta<\alpha \mid \beta \equiv 2(\bmod 3)\}| \leq \aleph_{1} .
\end{aligned}
$$


The following lemma will be a consequence of the forthcoming iteration lemmas.

3.1. Lemma. For each $\alpha \leq \kappa, \bar{P}_{\alpha}$ is $S$-closed and semiproper.

Assuming this lemma, the proof of Theorem $\mathrm{C}$ can be completed as follows: For $\alpha \leq \kappa$, define

$$
\begin{aligned}
\bar{P}_{\alpha}^{\prime}=\left\{p \in \bar{P}_{\kappa} \mid \forall \alpha \in \operatorname{supt}(p)(\alpha \equiv 1\right. & (\bmod 3) \vee \alpha \equiv 2(\bmod 3)) \\
& \left.\wedge \forall \beta \in \operatorname{supt}(p)\left(p(\beta) \text { is a } P_{\beta}^{\prime} \text {-name }\right)\right\} .
\end{aligned}
$$

$\left(p(\beta)\right.$ being a $P_{\beta}^{\prime}$-name, it only depends on $\dot{G}_{\beta} \cap P_{\beta}^{\prime}$.) Then any forcing extension via $\bar{P}_{\kappa}^{\prime}$ satisfies the conclusion of Theorem C:

Let $G^{\prime}$ be $\bar{P}_{\kappa}^{\prime}$-generic. It can be checked that the analogue of 2.4 holds in the new situation. In particular, there is a $G \bar{P}_{\kappa}$-generic such that $G \cap \bar{P}_{\kappa}^{\prime}=G^{\prime}$. With 3.1, the proof of 2.1 still works to show that in $V[G], \kappa=\omega_{2}$ and NS| $\mid \tilde{S}$ is $\kappa$-saturated. It then follows as before that in $V\left[G^{\prime}\right], \mathrm{NS} \mid \widetilde{S}$ is $\kappa$-saturated and $\mathscr{P}\left(\omega_{1}\right) / \mathrm{NS} \mid \widetilde{S}$ is a complete Boolean algebra.

By standard arguments $\bar{P}_{\kappa}^{\prime}$ has the $\kappa$-c.c., and the introduction of the collapses $\dot{R}_{\alpha}$ for $\alpha \equiv 2(\bmod 3)$ implies that in $V\left[G^{\prime}\right], \kappa=2^{\omega_{1}} \leq \omega_{3}$. But by 3.7 below, $\omega_{2}$ is preserved so that $\kappa=\omega_{3}$ in $V\left[G^{\prime}\right]$ and so the proof is complete.

The rest of this section is devoted to establishing 3.1 and the forthcoming 3.7. We build on Shelah [Sh2; Sh4, XIV] and refer to them for the more basic details about iterated forcing that are not provided in full.

For $\mu>\omega$, let $\mathscr{K}_{\mu}$ be the class of $\left\langle Q, \leq_{Q}, \leq_{Q}^{0}\right\rangle$ such that:

(i) $\left\langle Q, \leq_{Q}\right\rangle$ is a semiproper, $S$-closed notion of forcing, say with maximum element $\dot{\mathrm{i}}_{Q}$.

(ii) $\left\langle Q, \leq_{Q}^{0}\right\rangle$ is a poset so that: (a) $\leq_{Q}$ refines $\leq_{Q}^{0}$ (i.e. if $p \leq_{Q}^{0} q$, then $p \leq_{Q} q$ ); and (b) $\leq_{0}$ is $\mu$-closed (i.e. if $\left\langle p_{\alpha} \mid \alpha<\eta\right\rangle$ is $\leq_{Q}^{0}$-decreasing and $\eta<\mu$, then there is a $p \in Q$ such that $p \leq_{Q}^{0} p_{\alpha}$ for every $\left.\alpha<\eta\right)$.

We often suppress the subscripts $Q$ and furthermore identify $\left\langle Q, \leq_{Q}, \leq_{Q}^{0}\right\rangle$ with its domain $Q$ when there is little possibility of confusion. When we use forcing terminology for such a member of $\mathscr{K}_{\mu}$, we are referring to the $\left\langle Q, \leq_{Q}\right\rangle$ part.

Next, let $\mathscr{K}_{\mu}^{*}$ be the class of $\left\langle P_{\alpha}, \dot{Q}_{\alpha} \mid \alpha<\gamma\right\rangle$ where for each $\alpha<\gamma, P_{\alpha}$ is a notion of forcing, $\dot{Q}_{\alpha}$ is a $P_{\alpha}$-name and $\Vdash_{P_{\alpha}} \dot{Q}_{\alpha} \in \mathscr{K}_{\mu}$ and recursively:

(i) $P_{\alpha}$ consists of functions $p$ with domain $\alpha$ so that for each $\beta<\alpha, p(\beta)$ is a $P_{\beta}$-name such that $\Vdash p(\beta) \in \dot{Q}_{\beta}$, and setting

$$
\operatorname{supt}(p)=\left\{\beta<\alpha \mid \Vdash p(\beta) \neq \dot{\mathrm{i}}_{Q_{\beta}}\right\}
$$

as before,

$$
|\operatorname{supt}(p)| \leq \aleph_{1} \quad \text { and } \quad\left|\left\{\beta<\alpha \mid \neg\left(\Vdash p(\beta) \leq_{\dot{Q}_{\beta}}^{0} \dot{\mathrm{i}}_{Q_{\beta}}\right)\right\}\right| \leq \aleph_{0} .
$$

(ii) The ordering on $P_{\alpha}$ is given by:

$$
\begin{aligned}
& p \leq q \text { iff } \forall \beta<\alpha\left(p \mid \beta \Vdash p(\beta) \leq_{\dot{Q}_{\beta}} q(\beta)\right) \\
& \wedge\left|\left\{\beta \in \operatorname{supt}(p) \mid \neg\left(p \mid \beta \Vdash p(\beta) \leq_{\dot{Q}_{\beta}}^{0} q(\beta)\right)\right\}\right| \leq \aleph_{0} .
\end{aligned}
$$


If $P_{\gamma}$ is defined by taking $\alpha=\gamma$ in the above, we say that $P_{\gamma}$ is the iteration of $\left\langle P_{\alpha}, \dot{Q}_{\alpha} \mid \alpha<\gamma\right\rangle$. The introduction of the second partial order $\leq^{0}$ serves less to provide iteration lemmas of potentially wide applicability than to provide a uniform approach to 3.1. For that result, $\leq_{\dot{R}_{\alpha}}^{0}$ will coincide with $\leq_{\dot{R}_{\alpha}}$ when $\alpha \equiv 2(\bmod 3)$, i.e. when $\dot{R}_{\alpha}$ is the Levy collapse $\operatorname{col}\left(\omega_{2}, 2^{\omega_{2}}\right)$; and $\leq_{\dot{R}_{\alpha}}^{0}$ will just be equality for $\alpha \equiv 0(\bmod 3)$ and $\alpha \equiv 1(\bmod 3)$. Note then that by how we defined the $\bar{P}_{\alpha}$ 's from the $\dot{R}_{\alpha}$ 's, $\left\langle\bar{P}_{\alpha}, \dot{R}_{\alpha} \mid \alpha<\kappa\right\rangle \in \mathscr{K}_{\mu}^{*}$.

The usual iteration facts hold for members of $\mathscr{K}_{\mu}^{*}$ : e.g., if $\left\langle P_{\alpha}, \dot{Q}_{\alpha} \mid \alpha<\gamma\right\rangle \in$ $\mathscr{K}_{\mu}^{*}$, for any $\beta<\alpha<\gamma, P_{\beta} \ll P_{\alpha}$, i.e. every maximal antichain of $P_{\beta}$ is a maximal antichain of $P_{\alpha}$, and for the usual quotient poset $P_{\alpha} / P_{\beta}$ such that $P_{\alpha} \cong P_{\beta} * P_{\alpha} / P_{\beta}, P_{\alpha} / P_{\beta}$ is an iteration of a member of $\mathscr{K}_{\mu}^{*}$. To establish 3.1, we must verify that iterations in $\mathscr{K}_{\mu}^{*}$ preserve $S$-closure and semiproperness.

The following lemma provides the main induction step for establishing the preservation of $S$-closure:

3.2. Lemma. Suppose that $P_{\gamma}$ is the iteration of $\mathscr{P}=\left\langle P_{\alpha}, \dot{Q}_{\alpha} \mid \alpha<\gamma\right\rangle \in \mathscr{K}_{\mu}^{*}$ and $\delta<\eta \leq \gamma$. Then the following holds for sufficiently large regular $\lambda$ :

Assume that $N$ is countable with $\langle N, \in\rangle \prec\left(H_{\lambda}, \in\right\rangle, N \cap \omega_{1} \in S,\{\mathscr{P}, \delta, \eta\}$ $\subseteq N,\left\langle p_{n} \mid n \in \omega\right\rangle$ is a $P_{\eta}$-generic sequence for $N$, and $q \in P_{\delta}$ satisfies $q \leq p_{n} \mid \delta$ for every $n \in \omega$. Then there is a $q^{+} \in P_{\eta}$ such that $q^{+} \mid \delta=q$ and $q \leq p_{n}$ for every $n \in \omega$.

Proof. By adjusting names, we can assume for convenience in what follows that for each $n \in \omega$ and $\alpha<\eta$ :

(i) $p_{n+1} \mid \alpha \Vdash p_{n+1}(\alpha) \leq^{0} p_{n}(\alpha)$ iff $\Vdash p_{n+1}(\alpha) \leq^{0} p_{n}(\alpha)$, and

(ii) $\Vdash p_{n+1}(\alpha) \leq p_{n}(\alpha)$.

We now define a function $q^{+}$with domain $\eta$ as follows: Fix a well-ordering $W$ of sufficiently large $V_{\rho}$. Set $q^{+} \mid \delta=q$. For $\delta \leq \alpha<\eta$, having defined $q^{+} \mid \alpha$ for $\alpha<\eta$ so that recursively $q^{+} \mid \alpha \in P_{\alpha}$, define $q^{+}(\alpha)$ as follows:

(a) If for some $k \in \omega, q^{+} \mid \alpha \Vdash$ " $\left\langle p_{n}(\alpha) \mid k<n<\omega\right\rangle$ is $\leq^{0}$-descending in $\dot{Q}_{\alpha}-\left\{\dot{1}_{Q_{\alpha}}\right\}$ ", then since by definitions of $\mathscr{K}_{\mu}$ and $\mathscr{K}_{\mu}^{*}, \Vdash_{P_{\alpha}}$ " $\leq_{Q_{\alpha}}^{0}$ is $\mu$-closed" and $\mu>\omega$, there is a $P_{\alpha}$-name $\tau$ so that $q^{+} \mid \alpha \Vdash$ " $\tau$ is a $\leq 0$-lower bound for $\left\langle p_{n}(\alpha) \mid k<n<\omega\right\rangle$ ". Let $q^{+}(\alpha)$ be the $W$-least such $\tau$. Else:

(b) If for some $k \in \omega$ and $P_{\alpha}$-name $\tau, q^{+} \mid \alpha \Vdash$ " $\left\langle p_{n}(\alpha)\right| k\langle n<\omega\rangle$ is $\leq_{Q_{\alpha}}$-descending in $\dot{Q}_{\alpha}-\left\{1_{Q_{\alpha}}\right\}$ with $\tau$ a $\leq Q_{\alpha}$-lower bound", then let $q^{+}(\alpha)$ be the $W$-least such $\tau$. Otherwise:

(c) Set $q^{+}(\alpha)=\dot{1}_{Q_{\alpha}}$.

This definition perpetuates $q^{+} \mid \alpha \in P_{\alpha}$ for every $\alpha \leq \eta$ : Clearly,

$$
\left|\operatorname{supt}\left(q^{+} \mid \alpha\right)\right| \leq\left|\bigcup_{n} \operatorname{supt}\left(p_{n} \mid \alpha\right)\right| \leq \aleph_{1} \text {. }
$$

Also, conditions on $\leq^{0}$ and (i) above imply that there is a countable set $E$ such that for $\beta \in \alpha-E, \Vdash$ “ $p_{n+1}(\beta) \leq^{0} p_{n}(\beta) \leq^{0} \dot{\mathrm{i}}_{Q_{\beta}}$ ” for every $n \in \omega$. For such $\beta, q^{+}(\beta)$ was defined either through clause (a), or if not, neither through clause (b) as $\leq$ refines $\leq^{0}$, but through clause (c). But both (a) and (c) lead to $\Vdash q^{+}(\beta) \leq \mathrm{i}_{Q_{\zeta}}$, and so

$$
\left\{\beta<\alpha \mid \neg\left(\Vdash q^{+}(\beta) \leq^{0} \dot{\mathrm{i}}_{Q_{\beta}}\right)\right\} \subseteq E,
$$

confirming that $q^{+} \mid \alpha \in P_{\alpha}$. 
We next establish by induction on $\alpha \leq \eta$ that

(1) for every $n \in \omega, q^{+} \mid \alpha \Vdash q^{+}(\alpha) \leq p_{n}(\alpha)$.

(2) If $\alpha \notin N-\delta$, then for every $n \in \omega, q^{+} \mid \alpha \Vdash q^{+}(\alpha) \leq^{0} p_{n}(\alpha)$.

As $N$ is countable, this suffices to verify that $q^{+} \leq p_{n}$ for every $n \in \omega$ as desired. For $\alpha<\delta$, the results are immediate; what remains splits into two cases:

Case 1. $\delta \leq \alpha<\eta$ and $\alpha \in N$. Then $P_{\alpha}, \dot{Q}_{\alpha} \in N$, and we have $q\left|\alpha \leq p_{n}\right| \alpha$ for every $n \in \omega$ by induction. Since $\left\langle p_{n} \mid n \in \omega\right\rangle$ is a $P_{\eta}$-generic sequence for $N$, it follows first that $\left\langle p_{n}|\alpha| n \in \omega\right\rangle$ is a $P_{\alpha}$-generic sequence for $N$, and second that $q^{+} \mid \alpha \Vdash$ " $\left\langle p_{n}(\alpha) \mid n \in \omega\right\rangle$ is a $\dot{Q}_{\alpha}$-generic sequence for $\check{N}\left[\dot{G}_{\alpha}\right]$ ". But by definitions of $\mathscr{K}_{\mu}$ and $\mathscr{K}_{\mu}^{*}, \Vdash_{P_{\alpha}}$ " $\dot{Q}_{\alpha}$ is $S$-closed", and since $N \cap \omega_{1} \in S$, (a) or (b) of the definition of $q^{+}$applies at $\alpha$ and (1) follows. (2) holds vacuously.

Case 2. $\delta \leq \alpha<\eta$ and $\alpha \notin N$. The $\leq^{0}$ conditions on the $p_{n}$ 's imply that for each $n \in \omega$,

$$
E_{n}=\left\{\beta<\eta \mid \neg\left(\Vdash p_{n+1}(\beta) \leq^{0} p_{n}(\beta) \leq^{0} \dot{1}_{Q_{\beta}}\right)\right\}
$$

is countable, and clearly $E_{n} \in N$, so that $E_{n}$ is countable in $N$. Hence, $\bigcup_{n} E_{n} \subseteq N$, so that $\alpha \notin \bigcup_{n} E_{n}$. Hence, $q^{+}(\alpha)$ was defined either through clause (a), or if not, neither through clause (b) as $\leq$ refines $\leq^{0}$, but through clause (c). Both (1) and (2) now follow in this case also.

The following preservation result now follows in straightforward fashion:

3.3. Proposition. Suppose that $P_{\gamma}$ is the iteration of $\left\langle P_{\alpha}, \dot{Q}_{\alpha}\right| \alpha\langle\gamma\rangle \in \mathscr{K}_{\mu}^{*}$ and $\delta<\eta \leq \gamma$. Then $P_{\eta} / P_{\delta}$ is $S$-closed.

The following lemma provides the main induction step for establishing the preservation of semiproperness. Again, for a notion of forcing $P, \dot{G}_{P}$ denotes the canonical $P$-name for its generic object. For a set $M$ and $q \in P$, to say that $q$ decides $\dot{G}_{P} \cap \check{M}$ means of course that there is a set $A \in V$ such that $q \Vdash \check{A}=\dot{G}_{P} \cap \check{M}$. This happens for example if $M$ is countable and $q$ is a lower bound to a $P$-generic sequence for $M$, with $A=\{r \in P \cap M \mid q \leq r\}$. Finally, for $\lambda>\omega$ regular and $\langle N, \epsilon\rangle \prec\left\langle H_{\lambda}, \epsilon\right\rangle$ with $P \in N$, if $G$ is $P$-generic over $V$, then let $N[G]$ be the set of interpretations $\{\tau[G] \mid \tau \in N$ is a $P$-name $\}$. By Shelah [Sh2, p. 88],

$$
\langle N[G], \epsilon\rangle \prec\left\langle H_{\lambda}^{V[G]}, \epsilon\right\rangle .
$$

We let $N\left[G_{P}\right]$ be a canonical $P$-name for $N[G]$.

3.4. Lemma. Suppose that $P_{\gamma}$ is the iteration of $\mathscr{P}=\left\langle P_{\alpha}, \dot{Q}_{\alpha} \mid \alpha<\gamma\right\rangle \in \mathscr{K}_{\mu}^{*}$ and $\delta<\eta \leq \gamma$. Then the following holds for sufficiently large regular $\lambda$ :

Assume that $N$ and $M$ are countable with $\langle N, \epsilon\rangle \prec\langle M, \epsilon\rangle \prec\left\langle H_{\lambda}, \epsilon\right\rangle$, $\{\mathscr{P}, \delta, \eta\} \subseteq N, N \in M$, and $M \cap \omega_{1} \in S$. Assume also that $q \in P_{\delta}$ is $\left\langle N, P_{\delta}\right\rangle$-semigeneric, $q$ is $\left\langle M, P_{\delta}\right\rangle$-generic and decides $\dot{G}_{P_{\delta}} \cap \check{M}$, and $p \in P_{\eta} \cap N$ with $q \leq p \mid \delta$.

Then there is a $q^{+} \in P_{\eta}$ with $q^{+} \leq p$ and $q^{+} \mid \delta=q$ such that $q^{+}$is $\left\langle N, P_{\eta}\right\rangle-$ semigeneric and $q^{+}$is $\left\langle M, P_{\eta}\right\rangle$-generic and decides $\dot{G}_{P_{\eta}} \cap \check{M}$.

Proof. We establish this by induction on $\eta$, for all $\delta, N, M, p$, and $q$. 
For $\eta$ a successor, we can clearly assume that $\eta=\delta+1$. By definitions of $K_{\mu}$ and $\mathscr{K}_{\mu}^{*}, \Vdash_{P_{\delta}}$ " $\dot{Q}_{\delta}$ is semiproper", and so with $(*)$ just before 3.1 in mind, we have

$$
\Vdash_{P_{\delta}} \exists r \in \dot{Q}_{\delta}\left(r \text { is }\left\langle N\left[G_{P_{\delta}}\right], P_{\delta}\right\rangle \text {-semigeneric } \wedge r(\delta) \leq p(\delta)\right) .
$$

Since $N \in M$ and $q$ is $\left\langle M, P_{\delta}\right\rangle$-generic and decides $\dot{G}_{P_{\delta}} \cap \check{M}$, by (*) applied syntactically there is a $q^{\prime} \in P_{\delta} \cap M$ with $q \leq q^{\prime}$ and a $P_{\delta}$-name $r^{\prime} \in$ $M$ such that $q^{\prime}$ forces the assertion of $(* *)$ with $r^{\prime}$. Since $M \cap \omega_{1} \in S$, $\Vdash_{P_{\delta}}$ " $\dot{Q}_{\delta}$ is $S$-closed" by the definitions, and with $(*)$ in mind for $M$, it is now straightforward to find a $q^{+} \in P_{\eta}$ as desired, noting that in the sense of $\Vdash_{P_{\delta}}$, any lower bound to a $\dot{Q}_{\delta}$-generic sequence for $\check{M}$ decides $\dot{G}_{Q_{\delta}} \cap \check{M}$.

For $\eta$ a limit, let $\left\langle\beta_{n} \mid n \in \omega\right\rangle$ enumerate $M \cap\{\beta \mid \delta \leq \beta<\eta\}$ with $\beta_{0}=\delta$. Let $\left\{\dot{\tau}_{n}^{l}: n<\omega\right\}$ enumerate the $P_{\eta}$-names of ordinals belonging to $N$ if $l=1$ and $M$ if $l=2$. Define $\alpha_{n}, q_{n}, p_{n}$, and $N_{n}$ by induction on $n \in \omega$ so that henceforth writing $\dot{G}_{n}$ for $\dot{G}_{P_{\alpha_{n}}}$, we have:

(a) $\alpha_{0}=\delta, q_{0}=q, p_{0}=p$ and generally $q_{n} \in P_{\alpha_{n}}$ with $q_{n} \leq p \mid \alpha_{n}$ and $q_{n+1} \mid \alpha_{n}=q_{n}, p_{n} \in N\left[\dot{G}_{n}\right] \cap P_{\alpha_{n}}\left(\subseteq M \cap P_{\alpha}\right), p_{n+1} \leq p_{n}$, and $p_{n+1}$ decides a value for $\dot{\tau}_{n}$.

(b) $q_{n}$ is $\left\langle N, P_{\alpha_{n}}\right\rangle$-semigeneric, and $q_{n}$ is $\left\langle M, P_{\alpha_{n}}\right\rangle$-generic and decides $\dot{G}_{n} \cap \check{M}$.

(c) $q_{n} \Vdash \alpha_{n+1}=\max \left\{\left\{\beta_{k} \mid k \leq n\right\} \cap N\left[\dot{G}_{n}\right]\right\}$.

(Note for (c) that if $q_{n}$ decides $\dot{G}_{n} \cap \dot{M}$ by (b), then $N \in M$ implies that $q_{n}$ decides $\dot{G}_{n} \cap \check{N}$ and hence $N\left[\dot{G}_{n}\right]$.)

The case $n=0$ follows from our initial assumption. Suppose now that $q_{n}$ has already been defined. By $(*)$ just before 3.4 applied syntactically, $\Vdash$ “ $N\left[\dot{G}_{n}\right] \prec M\left[\dot{G}_{n}\right] \prec\left\langle H_{\lambda}^{V\left[\dot{G}_{n}\right]}, \in\right\rangle$ ”. Moreover, since $q_{n}$ is $\left\langle M, P_{\alpha_{n}}\right\rangle$-generic, we have $q_{n} \Vdash M\left[\dot{G}_{n}\right] \cap \omega_{1}=\check{M} \cap \omega_{1} \in \check{S}$. Hence, with $\alpha_{n+1}$ as stipulated by (c) it is straightforward to apply the induction hypothesis in the sense of $q_{n} \Vdash$ and then to find an appropriate $q_{n+1} \in P_{\alpha_{n+1}}$ as desired. There is no problem in defining $p_{n+1}$.

We can now define a $q^{+} \in P_{\eta}$ so that $\operatorname{supt}\left(q^{+}\right)=\bigcup_{n} \operatorname{supt}\left(q_{n}\right)$, and for any $\beta$ in this set, $q^{+}(\beta)=q_{n}(\beta)$ for some (any) $n$ such that $\beta \in \operatorname{supt}\left(q_{n}\right)$. As in [Sh2, Sh4], $q \leq p_{n}$ so $q^{+}$is $\left\langle N, P_{\alpha}\right\rangle$-semigeneric. $q^{+}$is not necessarily $\left(M, P_{\alpha}\right)$ generic, but its existence shows that there is $q^{\prime} \in P_{\eta} \cap M, q^{\prime} \mid \alpha \leq q^{+}, q^{\prime}$ is $\left\langle N, P_{\alpha}\right\rangle$-semigeneric. Now we can find a $q^{+}$really as required.

The following preservation result is now clear, since for $\langle N, \epsilon\rangle \prec\left(H_{\lambda}, \epsilon\right\rangle$ as in 3.4, we can always find a countable $M$ such that $N \in M,\langle M, \in\rangle \prec\left\langle H_{\lambda}, \in\right\rangle$, and $M \cap \omega_{1} \in S$ by the stationariness of $S$.

3.5. Proposition. Suppose that $P_{\gamma}$ is the iteration of $\left\langle P_{\alpha}, \dot{Q}_{\alpha} \mid \alpha<\gamma\right\rangle \in \mathscr{K}_{\mu}^{*}$ and $\delta<\eta \leq \gamma$. Then $P_{\eta} / P_{\delta}$ is semiproper.

Proof of 3.1. By 3.3 and 3.5 we get $S$-completeness and semiproperness.

Finally, we establish the preservation of $\omega_{2}$ in a special case; a similar result appears in Shelah [Sh2, VIII, §1].

3.6. Proposition. Suppose that $\mathrm{CH}$ holds, and $P$ is the iteration of $\mathscr{P}=$ $\left\langle P_{\alpha}, \dot{Q}_{\alpha} \mid \alpha<\gamma\right\rangle \in \mathscr{K}_{\omega_{2}}^{*}$ where for each $\alpha<\gamma, \Vdash_{P_{\alpha}}{ }^{\prime \prime} \leq_{\dot{Q}_{a}}^{0}=\leq_{\dot{Q}_{\alpha}}$ and $Q_{\alpha}$ sat- 
isfies $\aleph_{2}$-pic" (see [Sh2, VII, Definition 2]) or $\Vdash_{P_{\alpha}}$ “ $\leq_{\dot{Q}_{\alpha}}^{0}$ is the equality relation on $\dot{Q}_{\alpha}$." Then forcing with $P$ preserves $\omega_{2}$.

Proof. Assume to the contrary that for some $p \in P$ and $P$-name $\tau, p \Vdash$ " $\tau: \check{\omega}_{1} \rightarrow \check{\omega}_{2}$ is a bijection". Taking a regular $\lambda$ sufficiently large, we proceed by induction on $\xi<\omega_{2}$ to define $p_{\xi} \in P$ so that $\xi<\zeta<\omega_{2}$ implies that for every $\alpha<\gamma$, $p_{\zeta}(\alpha) \leq{ }^{0} p_{\xi}(\alpha)$, and accompanying $q_{\xi} \leq p_{\xi}, N_{\xi}$, and $A_{\xi}$ as follows:

Set $p_{0}=p$. At limits $\zeta<\omega_{2}$ with $p_{\xi}$ having been defined for $\xi<\zeta$, by the $\omega_{2}$-closure of the $\leq_{\dot{Q}_{\alpha}}^{0}$ 's, let $p_{\zeta} \in P$ be such that for every $\xi<\zeta$ and $\alpha<\gamma$, $\Vdash p_{\zeta}(\alpha) \leq^{0} p_{\xi}(\alpha)$.

To handle the successor stage, suppose that $p_{\xi}$ is given. First let $N_{\xi}$ be countable with $\left\langle N_{\xi}, \epsilon\right\rangle \prec\left\langle H_{\lambda}, \epsilon\right\rangle,\left\{\mathscr{P}, \xi, p_{\xi}\right\} \subseteq N_{\xi}$, and $N_{\xi} \cap \omega_{1} \in S$. Then define $q_{\xi} \leq p_{\xi}$ by first choosing a $P$-generic sequence for $N_{\xi}$ starting with $p_{\xi}$ and then using the clauses (a), (b), and (c) as in the proof of 3.2. Hence $q_{\xi}$ is $\left\langle N_{\xi}, P\right\rangle$-generic and setting $A_{\xi}=\left\{r \in P \cap N_{\xi} \mid q_{\xi} \leq r\right\}$, we have $q_{\xi} \Vdash \check{A}_{\xi}=$ $\dot{G}_{P} \cap \check{N}_{\xi}$. We can assume that $\Vdash q_{\xi}(\alpha) \leq{ }^{0} p_{\xi}(\alpha)$ iff $q_{\xi} \mid \alpha \Vdash q_{\xi}(\alpha) \leq^{0} p_{\xi}(\alpha)$; similarly for $\leq$; and also that each $q_{\xi}(\alpha)$ depends only on $p_{\xi}(\alpha)$ and not on $\xi$ (by carrying out the entire procedure canonically according to some wellordering of a sufficiently large $\left.V_{\rho}\right)$. Finally, define $p_{\xi+1}$ as follows:

$$
p_{\xi+1}(\alpha)= \begin{cases}q_{\xi}(\alpha) & \text { if } \Vdash q_{\xi}(\alpha) \leq^{0} p_{\xi}(\alpha), \\ p_{\xi}(\alpha) & \text { otherwise. }\end{cases}
$$

Proceeding with the proof, a straightforward $\Delta$-system argument with $\mathrm{CH}$ shows that there are $\xi_{0}<\xi_{1}<\omega_{2}$ such that: $N_{\xi_{0}} \cap \omega_{1}=N_{\xi_{1}} \cap \omega_{1}$, and there is an isomorphism $h:\left\langle N_{\xi_{0}}, \in, A_{\xi_{0}}\right\rangle \rightarrow\left\langle N_{\xi_{1}}, \in, A_{\xi_{1}}\right\rangle$ with $h \mid\left(N_{\xi_{0}} \cap N_{\xi_{1}}\right)$ the identity, $h(\mathscr{P})=\mathscr{P}, h\left(\xi_{0}\right)=\xi_{1}$, and $h\left(p_{\xi_{0}}\right)=p_{\xi_{1}}$. By assumption, there is an $\eta \in N_{\xi_{0}} \cap \omega_{1}$ such that $q_{\xi_{0}} \Vdash \dot{\tau}(\eta)=\xi_{0}$. Hence, $\exists r \in A_{\xi_{0}}\left(r \Vdash \dot{\tau}(\eta)=\xi_{0}\right)$. Applying $h$, we have $\exists r \in A_{\xi_{1}}\left(r \Vdash \dot{\tau}(\eta)=\xi_{1}\right)$ since $h$ is the identity on $N_{\xi_{0}} \cap \omega_{1}$. Consequently, $q_{\xi_{1}} \Vdash \dot{\tau}(\eta)=\xi_{1}$. However, it is not difficult to check that $q_{\xi_{0}}$ and $q_{\xi_{1}}$ are compatible, reaching a contradiction:

The definitions of $q_{\xi_{0}}$ and $q_{\xi_{1}}$ as in the proof of 3.2 show that the countable sets

$$
E_{i}=\left\{\alpha \in \operatorname{supt}\left(q_{\xi_{i}}\right) \mid \neg\left(\Vdash q_{\xi_{i}}(\alpha) \leq^{0} p_{\xi_{i}}(\alpha)\right)\right\}
$$

for $i<2$ are such that $E_{i} \in N_{\xi_{i}}$ and $E_{i}$ is countable in $N_{\xi_{i}}$. We now argue that $\Vdash$ " $q_{\xi_{0}}(\alpha)$ and $q_{\xi_{1}}(\alpha)$ are compatible" by cases, depending on whether $\alpha \in \gamma-E_{0}, \alpha \in E_{0}-E_{1}$, or $\alpha \in E_{0} \cap E_{1}$ : If $\alpha \in \gamma-E_{0}$, then $\Vdash q_{\xi_{1}}(\alpha) \leq$ $p_{\xi_{1}}(\alpha) \leq^{0} p_{\xi_{0}+1}(\alpha) \leq 0 q_{\xi_{0}}(\alpha)$. If $\alpha \in E_{0}-E_{1}$, then $\Vdash q_{\xi_{0}}(\alpha) \leq p_{\xi_{0}}(\alpha)$ and $\alpha \in E_{0}$ implies that we are in the case $\Vdash_{P_{\alpha}}$ " $\leq_{\dot{Q}_{a}}^{0}$ is equality" of our assumption about $P$. Consequently, $\alpha \notin E_{1}$ implies that $\Vdash q_{\xi_{1}}(\alpha)=p_{\xi_{1}}(\alpha)=p_{\xi_{0}}(\alpha)$, and so we have $\Vdash q_{\xi_{0}}(\alpha) \leq q_{\xi_{1}}(\alpha)$. Finally, if $\alpha \in E_{0} \cap E_{1}$, then $\alpha \in N_{\xi_{0}} \cap N_{\xi_{1}}$ so the first case in the proposition occurs and we apply " $\aleph_{2}$-pic". This completes the proof.

In 3.6 we can combine the two possibilities to one as implicit in the proof.

3.7. Proposition. $P_{\kappa}^{\prime}$ (from the proof of Theorem $\mathrm{C}$ ) preserves $\omega_{2}$.

Proof. Let $\left\langle P_{\alpha}^{1}, \dot{Q}_{\alpha}^{1}: \alpha<\kappa\right\rangle \in \mathscr{K}_{\mu}$ be as above except that for $\alpha \equiv 0(\bmod 3)$, $\dot{Q}_{\alpha}^{1}$ is the trivial forcing (and if $\left.\alpha \not \equiv 0(\bmod 3), \dot{Q}_{\alpha}^{1}=\dot{R}_{\alpha}\right)$. Essentially, $P_{\alpha}^{1}=$ 
$P_{\alpha}^{\prime}$, and hence it suffices to prove that $P_{\alpha}^{1}$ preserves $\omega_{2}$. Now the assumption of 3.6 clearly holds for $\left\langle P_{\alpha}^{1}, \dot{Q}_{\alpha}^{1}: \alpha<\kappa\right\rangle$ (for $\aleph_{2}$-pic-see the discussion of $Q_{S}$ for more).

This finally completes the proof of Theorem C.

Instead of $2^{\omega_{1}}=\omega_{3}$, for any regular $\nu$ such that $\omega_{2} \leq \nu<\kappa$, it is possible to arrange $2^{\omega_{1}}=\nu^{+}=\kappa$ using $\mathscr{K}_{\nu}^{*}$ with $|\operatorname{supt}(p)| \leq \nu$ is place of $|\operatorname{supt}(p)| \leq$ $\omega_{1}$ in its definition.

\section{REFERENCES}

[B] James Baumgartner, Iterated forcing, Surveys in Set Theory (A. Mathias, ed.), London Math. Soc. Lecture Note Series, no. 87, Cambridge Univ. Press, 1983, pp. 1-59.

[BT] James Baumgartner and Alan Taylor, Saturation properties of ideals in generic extensions. II, Trans. Amer. Math. Soc. 27 (1982), 587-609.

[FMS] Matthew Foreman, Menachem Magidor, and Saharon Shelah, Martin's Maximum, saturated ideals and non-regular ultrafilters. Part I, Ann. of Math. (2) 127 (1988), 1-47.

[GS] Moti Gitik and Saharon Shelah, Cardinal preserving ideals, J. Symbolic Logic (to appear).

[J] Thomas Jech, Set theory, Academic Press, New York, 1978.

[Ke] Jussi Ketonen, Some combinatorial principles, Trans. Amer. Math. Soc. 188 (1974), 387-394.

[Ky] Kenneth Kunen, Some applications of iterated ultrapowers in set theory, Ann. Math. Logic 1 (1970), 179-227.

[P] R. S. Pierce, Distributivity in Boolean algebras, Proc. Amer. Math. Soc. 7 (1957), 983-992.

[Sh1] Saharon Shelah, A weak generalization of MA to higher cardinals, Israel J. Math. 30 (1978), 297-306.

[Sh2] __ Proper forcing, Lecture Notes in Math., vol. 940, Springer-Verlag, Berlin, 1986.

[Sh3] _ Iterated forcing and normals ideals on $\omega_{1}$, Israel J. Math. 60 (1987), 345-380.

[Sh4] _ Proper and improper forcing, Springer-Verlag, Berlin (to appear); revised and expanded version of [Sh2] .

[Si] Roman Sikorski, On an unsolved problem from the theory of Boolean algebras, Colloq. Math. 2 (1949), 27-29.

[ST] Edgar Smith and Alfred Tarski, Higher degrees of distributivity and completeness in Boolean algebras, Trans. Amer. Math. Soc. 84 (1957), 230-257.

[So] Robert Solovay, Real-valued measurable cardinals, Axiomatic Set Theory (Dana Scott, ed.), Proc. Sympos. Pure Math., vol. 13, part 1, Amer. Math. Soc., Providence, RI, 1971, pp. 397-428.

Department of Mathematics, Boston University, Boston, Massachusetts 02215-2411

E-mail address: aki@math.bu.edu

Department of Mathematics, The Hebrew University, Jerusalem, Israel 